\title{
Narrowband terahertz generation with chirped-and-delayed laser pulses in periodically poled lithium niobate
}

\author{
Frederike Ahr ${ }^{1,2,},{ }^{*}$ Spencer W. Jolly ${ }^{3,4}$, Nicholas H. Matlis ${ }^{1}$, Sergio Carbajo ${ }^{1}$, Tobias

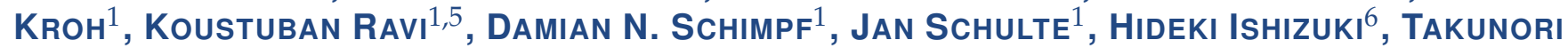 \\ TAIRA $^{6}$, Andreas R. MAIER ${ }^{4}$, ANd Franz X. KÄRTNER ${ }^{1,2,5}$ \\ ${ }^{1}$ Deutsches Elektronen Synchrotron (DESY) \& Center for Free-Electron Laser Science, Notkestrasse 85, 22607 Hamburg, Germany \\ ${ }^{2}$ Department of Physics, University of Hamburg, Luruper Chaussee 149, 22761 Hamburg, Germany \\ ${ }^{3}$ Institue of Physics of the ASCR, ELI-Beamlines project, Na Slovance 2, 18221 Prague, Czech Republic \\ ${ }^{4}$ Center for Free-Electron Laser Science \& Department of Physics, University of Hamburg, Luruper Chaussee 149, 22761 Hamburg, Germany \\ ${ }^{5}$ Department of Electrical Engineering and Computer Science, Research Laboratory of Electronics, Massachusetts Institute of Technology, Cambridge, MA \\ 02139, USA \\ ${ }^{6}$ Laser Research Center, Institute for Molecular Science, 38 Nishigonaka, Myodaiji, Okazaki 444-8585, Japan \\ *Corresponding author: frederike.ahr@desy.de
}

Compiled May 11, 2017

We generate narrowband terahertz $(\mathrm{THz})$ radiation in periodically poled lithium niobate (PPLN) crystals using two chirped-and-delayed driver pulses from a highenergy Ti:sapphire laser. The generated frequency is determined by the phase matching condition in the PPLN and influences the temporal delay of the two pulses for efficient terahertz generation. We achieve internal conversion efficiencies up to $0.13 \%$ as well as a record multi-cycle $\mathrm{THz}$ energy of $40 \mu \mathrm{J}$ at $0.544 \mathrm{THz}$ in a cryogenically-cooled PPLN. @ 2017 Optical Society of America

OCIS codes: (230.6080) Sources; (320.7110) Ultrafast nonlinear optics; (260.3090) Infrared, far; (160.3730) Lithium niobate;

http://dx.doi.org/10.1364/ao.XX.XXXXXX

The last couple of decades have seen a tremendous surge in development of terahertz (THz) sources of high energy and highpeak field for applications ranging from linear and nonlinear THz spectroscopy[1], to compact, THz-based electron acceleration [2]. For spectroscopic applications, the low energy of $\mathrm{THz}$ photons enables non-ionizing time-resolved studies of material properties [3], while for electron accelerators, the millimeter scale of $\mathrm{THz}$ radiation offers the advantages of compactness and high-acceleration gradients which are beneficial for driving ultrashort $\mathrm{X}$-ray sources. Optically generated $\mathrm{THz}$ pulses based on difference frequency generation (DFG) are exceptionally promising for these applications due to the high efficiencies, high peak fields and high degree of tunability they offer. With the development of the tilted pulse-front technique [4], percentlevel optical-to-THz conversion efficiencies have been achieved - using Fourier limited broadband near-infrared (NIR) drivers incident on bulk lithium niobate - enabling generation of single- cycle pulses in the mJ-range. By comparison, the performance of equivalent sources of multi-cycle $\mathrm{THz}$ pulses has lagged behind. Multi-cycle pulses, which have narrower bandwidths and longer pulse durations in the order of tens of picoseconds, offer complementary parameters relative to single-cycle pulses, and are advantageous for applications such as driving linear accelerator [2] and tuned excitation of specific material transitions [5]. Recent work in $\mathrm{THz}$ generation has thus seen a greater emphasis on development of efficient multi-cycle sources.

Among the primary challenges are achieving high conversion efficiencies and scaling to high energies. High efficiencies in nonlinear optical conversion processes require high incident optical intensities and management of the phase mismatch between optical and $\mathrm{THz}$ waves, which tends to be large in most nonlinear materials due to differences in the linear index for the two waves. Quasi-phase matching in PPLN has emerged [6-8] as a promising candidate for phase-matching in narrowband $\mathrm{THz}$ generation due to the large second order susceptibility, $\chi_{2}$, of lithium niobate. Recently, conversion efficiencies up to $0.12 \%$ and THz energies of $\sim 1 \mu \mathrm{J}$ were demonstrated by using Fourierlimited femtosecond pump pulses with optimized bandwidths and by using cryogenic cooling to minimize $\mathrm{THz}$ absorption in the PPLN crystal [9]. To produce the mJ-level THz pulses needed for acceleration applications [2], however, pump pulses in the range of 1 Joule are required. But scaling to Joule energies with short pump pulses is problematic because the incident energy is limited by the optically-induced damage threshold and by the limited aperture of commercially available PPLNs. A solution is thus to increase the pump pulse duration to allow greater energy to be carried without exceeding the intensity threshold for optical damage.

Temporally stretching of the NIR pump, as proposed in $[8,10]$, offers one method to increase the pulse duration and therefore scale up the pump-pulse energy. A side effect of chirping, how- 
ever, is that the instantaneous spectral content of the optical pulse is reduced, limiting the nonlinear conversion process that requires simultaneous presence of optical photons with frequencies separated by the frequency of the generated THz. This effect can be compensated by adding a second chirped pulse with an appropriate delay to provide the required instantaneous spectral content.

The chirp-and-delay concept was first described in 1994 to produce tunable, narrowband $\mathrm{THz}$ waves via photoconductive antennas [11]. Chirp-and-delay has been used to produce higher energy pulses of $10 \mu \mathrm{J}$ [12] in a hybrid approach employing the tilted pulse front technique. These pulses contained around 10 optical cycles and are thus not ideal for applications requiring very narrow-band radiation, like electron acceleration. Here, we demonstrate use of chirp-and-delay in combination with a periodically poled medium for the first time and generate record energies of narrow-band multi-cycle $\mathrm{THz}$ radiation. We investigate PPLN with varying poling periods and crystal lengths and study the effect of cryogenic cooling on the conversion efficiency.

DFG requires the presence of two distinct frequency components overlapped in space and time. These components can be provided by a single pulse of sufficiently large bandwidth or by two narrow-band pulses with distinct central frequencies. In the chirp-and-delay concept, two narrow-band components are provided by broadband but chirped pulses combined with well-defined temporal delay, $\Delta t$, as schematically illustrated in Fig. 1a. In this case, the instantaneous angular frequency of each pulse varies linearly in time, but the instantaneous angular frequency difference, $\Delta \omega$, is fixed by the delay and the chirp rate as shown in Fig. 1b. (a)

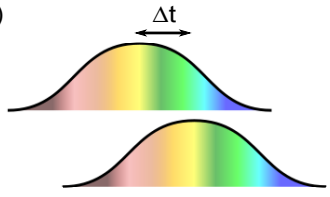

(b)

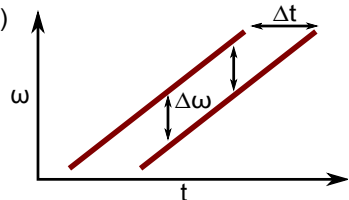

Fig. 1. a) Conceptual temporal arrangement of collinear chirpand-delay pulses. b) Wigner-plot illustrating the spectral content of the chirp-and-delay pulses vs. time.

The delay can then be tuned so that $\Delta \omega\left(\Delta t_{\text {opt }}\right)=\Omega$, where $\Delta t_{\text {opt }}$ is the optimal delay to achieve the desired THz frequency $\Omega$. Assuming a second order spectral phase coefficient, i.e. group-delay dispersion (GDD), of $\phi_{2}$, which is equivalent to a linear chirp rate of $1 / \phi_{2}$, the optimum delay is

$$
\Delta t_{o p t}=\Omega \phi_{2} .
$$

Optimizing $\mathrm{THz}$ generation in periodically poled crystals requires $\Delta \omega$ to be tuned to the phase-matched $\mathrm{THz}$ frequency, $\nu_{\mathrm{THz}}=\Omega / 2 \pi$, which is primarily determined by the poling period according to

$$
\Omega=\frac{2 \pi c}{\Lambda\left|n(\Omega)-n_{\mathrm{g}}\right|},
$$

with $c$ the speed of light, $\Lambda$ the poling period, and $n(\Omega)$ and $n_{\mathrm{g}}$ are the phase and group refractive indices for the THz and IR waves respectively $[13,14]$. To a lesser extent, $\Omega$ is also determined by the temperature through the temperature-dependent indices of refraction $[9,15]$. The relative THz bandwidth, $\Delta \Omega / \Omega$, and hence the number of cycles generated, $N$, is decreasing with the crystal length $L[8,16]$. By contrast, in narrowband $\mathrm{THz}$ generation with bulk lithium niobate, where direct phase matching via a tilted pulse-front pumping takes place, the $\mathrm{THz}$ pulse length, and thus the number of optical cycles, is directly defined by the IR pulse duration [12].

Experiments were done using the ANGUS 200 TW double CPA, Ti:Sa-based laser system providing $5 \mathrm{~J}$ pulses at $5 \mathrm{~Hz}$, compressible to $25 \mathrm{fs}$. The spectrum was centered at $800 \mathrm{~nm}$ with a $35 \mathrm{~nm}$ FWHM bandwidth, resulting in chirped pulses of $260 \mathrm{ps}$ FWHM, duration and GDD of $\phi_{2}=2.3 \mathrm{ps}^{2}$ before compression.

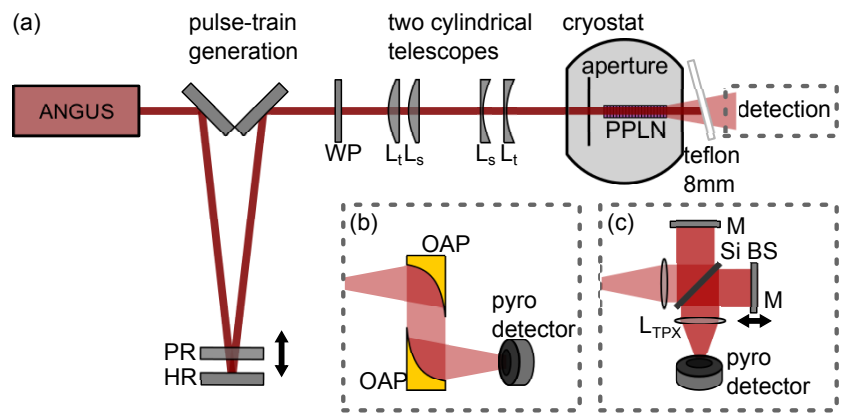

Fig. 2. a) Chirp-and-delay setup using a combination of partial reflector (PR) and high reflector (HR) to generate a chirped pulse pair plus lower-energy trailing pulses. b) THz pulse energy measurement setup. c) $\mathrm{THz}$ frequency measurement setup based on Michelson interferometer. KEY: WP: half wave plate; $\mathrm{L}_{s}$ : sagittal lens; $\mathrm{L}_{t}$ : tangential lens; OAP: off-axis parabola; $\mathrm{L}_{\mathrm{TPX}}$ : TPX lens; M: Ag mirror; Si BS: Si beam splitter.

A train of pulses of identical, nearly linear chirp and tunable delay was created from the uncompressed beam by using a $38 \%$ partial reflector (PR) mounted on a delay stage with the PR coating facing a high reflector (HR), as shown in Fig 2a. This combination resulted in two pump pulses of approximately equal intensity followed by a series of pulses with significantly lower energies originating from the multiple reflections. The energy of the pulses was tuned using a waveplate and two thin film polarizers (not shown), while a second half-waveplate (WP) matched the polarization of the chirped-and-delayed pulses to the optical axis of the PPLN. Two cylindrical telescopes matched the beam shape to the clear aperture of the PPLN: the tangential and sagittal telescopes reduce the vertical and horizontal beam size to $3 \mathrm{~mm}$ and $10 \mathrm{~mm}$, respectively to match the aperture of the PPLN. The PPLN was mounted in a cryostat for cryogenic cooling using liquid nitrogen to reduce $\mathrm{THz}$ absorption in the crystal. A ceramic aperture installed inside the cryostat just in front of the PPLN input surface mitigated the heat load and acted as a mask to shape the beam and ensure it wasn't clipped by the crystal. The entrance and exit ports of the cryostat were fused silica windows anti-reflection (AR) coated for $800 \mathrm{~nm}$. The transmission ratio of the windows for $\mathrm{THz}$ pulses was calibrated independently, and was found to be $80 \%$ in our frequency range.

THz was generated using PPLN crystals of four different poling periods: $\Lambda=125 \mu \mathrm{m}, 212 \mu \mathrm{m}, 330 \mu \mathrm{m}$, and $400 \mu \mathrm{m}$, obtained from HC Photonics (excluding the PPLN at $330 \mu \mathrm{m}$, which is provided by Prof. T. Taira). To measure the THz pulse energy, two 2 " gold-coated off-axis parabolas of 4 " focal length were used to collimate the $\mathrm{THz}$ and focus it onto a pyro-detector (Gentec-EO: THZ9B-BL-BNC). In an interchangeable setup, the frequency of the $\mathrm{THz}$ was measured using a Michelson interferometer with a $3.5 \mathrm{~mm}$ thick silicon wafer as a beamsplitter and a variable delay on one arm. The $\mathrm{THz}$ was collimated into the interferometer 
using a $100 \mathrm{~mm}$ focal length TPX lens. The interference pattern was then determined by focusing the THz with a second TPX lens onto the pyro-detector and scanning the variable delay. An $8 \mathrm{~mm}$ thick teflon plate ( $\mathrm{THz}$ transmission of $80 \%$ ) was used to dump the IR pump before the $\mathrm{THz}$ detection setups and to allow measurements of the transmitted optical spectra using an HR4000 fiber spectrometer (Ocean Optics).

In order to verify the chirp-and-delay mechanism we measured the dependence of the optimum delay on the frequency of the generated $\mathrm{THz}$, which, from Eq. 1, we expect to be linear. The $\mathrm{THz}$ frequency was varied by using crystals of different poling period, and the $\mathrm{THz}$ output was measured as a function of the delay, (by scanning the position of the PR), for each of the four poling periods (Fig. 3). Panel 3a) shows that the optimum delay depends inversely on the poling period, as expected from Eqns. 1 \& 2. We note the development of additional substructure for higher delays, which we attribute to the presence of residual third-order dispersion in the chirped pulses and the presence of a pulse train from the HR/PR combination rather than the ideal case of two isolated pulses. A detailed analysis of these effects has been done, but is beyond the scope of this work and will be presented elsewhere [17].
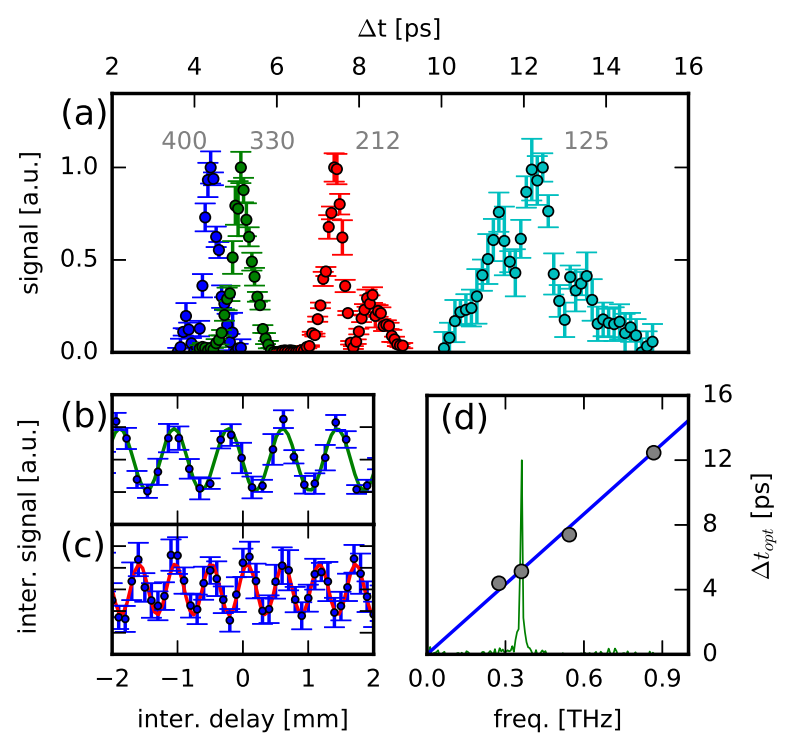

Fig. 3. a) Normalized $\mathrm{THz}$ signal vs. delay for PPLN poling periods of $400 \mu \mathrm{m}, 330 \mu \mathrm{m}, 212 \mu \mathrm{m}$ and $125 \mu \mathrm{m}$. b) \& c) Interferometer data (dots) and sinusoidal fits (lines) for crystals of poling period $330 \mu \mathrm{m}$ and $212 \mu \mathrm{m}$ yielding $\mathrm{THz}$ wavelengths of $0.83 \mathrm{~mm}$ and $0.55 \mathrm{~mm}$, respectively. d) Comparison of measured (grey dots) and predicted (blue line) optimum delays for four poling periods. The Fourier transform of the data in b) is shown in the background.

The connection between the poling period and the $\mathrm{THz}$ frequency is well established, and has been measured previously [9]. For completeness, however, the frequency of the THz generated by the $330 \mu \mathrm{m}$ and $212 \mu \mathrm{m}$ poling period crystals was measured using the interferometer. These crystals were chosen for interferometry since they produce the highest signal due to frequency scaling of absorption and the DFG process. Figures $3 b \& c$ are showing these interferometic traces, which are subsets of longer measurements, that show greater than 60 cycles for the $330 \mu \mathrm{m}$ PPLN. Using sinusoidal fits, THz wavelengths of
$0.83 \mathrm{~mm}$ and $0.55 \mathrm{~mm}$, corresponding to frequencies of $0.361 \mathrm{THz}$ and $0.544 \mathrm{THz}$ were determined, in good agreement with the values of $0.341 \mathrm{THz}$ and $0.532 \mathrm{THz}$, respectively, predicted by Eq. 2 and using refractive indices from [18]. For the $125 \mu \mathrm{m}$ and $400 \mu \mathrm{m}$ poling period crystals, which produced smaller $\mathrm{THz}$ signals, we used values for the frequencies of $0.867 \mathrm{THz}$ and $0.275 \mathrm{THz}$ respectively, which we measured using the identical crystals in a previous work [9].

The dependence of the optimal delay on $\mathrm{THz}$ frequency is plotted in Figure 3d. The measured delays (grey dots) agree well with the prediction based on Eq. 1 and using the GDD of the ANGUS system, verifying the chirp-and-delay mechanism. For example, the $330 \mu \mathrm{m}$ poling period crystal had a measured optimum delay of $\Delta t=5.13 \mathrm{ps}$ in very good agreement with the predicted delay of $5.22 \mathrm{ps}$ at $0.361 \mathrm{THz}$. The narrowness of the $\mathrm{THz}$ spectrum was verified by taking a Fourier transform of the $330 \mu \mathrm{m}$ interferometry data, shown in the background of Figure $3 \mathrm{~d}$. The $212 \mu \mathrm{m}$ data set was not long enough to produce an accurate spectrum and so was not plotted.

The chirp-and-delay scheme was then optimized by studying the $\mathrm{THz}$ output as a function of the incident pump fluence for varying crystal temperatures and interaction lengths. To distinguish between the intrinsic performance of the scheme and engineering factors such as transport of photons to and from the crystal or Fresnel losses at the surfaces, the performance was characterized using two primary metrics: 1 ) the internal conversion efficiency (hereafter, "efficiency"), defined as the ratio of the THz energy before exiting the crystal rear surface and the IR input energy after entering the crystal front surface and 2) the $\mathrm{THz}$ energy extracted from the crystal. A so-called extracted efficiency in this case would be 2.1 times lower than the internal efficiency due to Fresnel losses. Figure 4 shows the conversion efficiency vs. incident pump fluence at room and cryogenic temperatures for two crystal lengths of $212 \mu \mathrm{m}$-poled PPLN.

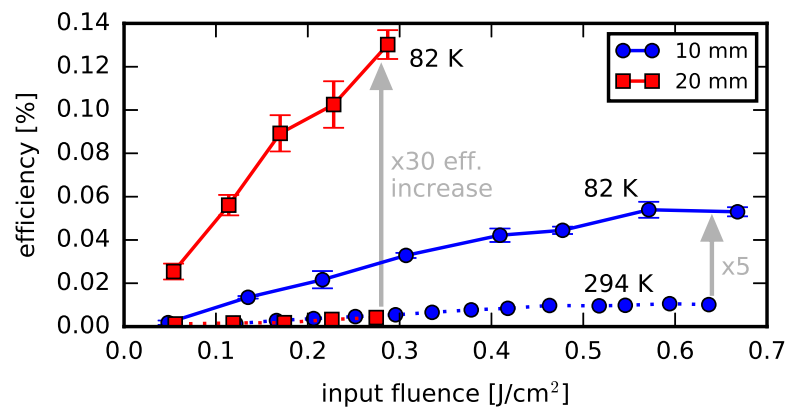

Fig. 4. Conversion efficiency of $212 \mu \mathrm{m}$ poling period PPLNs at room- (dotted) and cryogenic (solid) temperature as a function of pump fluence, showing improvement with cooling.

At room temperature $(294 \mathrm{~K})$, the efficiency is nearly identical for the $10 \mathrm{~mm}$ and $20 \mathrm{~mm}$ long crystals. This can be explained by the large absorption coefficient of lithium niobate $\left(5.2 \mathrm{~cm}^{-1}\right.$ [18]) at room temperature which limits the effective interaction length to only a few millimeters for both crystals $[6,9]$. By cooling the crystals to $82 \mathrm{~K}$ the absorption coefficient was reduced to $2.8 \mathrm{~cm}^{-1}$ [18], which significantly increased the effective interaction length, allowing greater advantage to be taken of longer crystals and thus improving the conversion efficiency. Compared to room temperature, the cryo-cooled $10 \mathrm{~mm}$ long crystal showed a five-fold increase in conversion efficiency, while the 
$20 \mathrm{~mm}$ long crystal showed a 30-fold increase, demonstrating the compounding benefits of reducing the $\mathrm{THz}$ absorption. For the $10 \mathrm{~mm}$ crystal, the efficiency saturated at a value of $0.054 \%$ at a pump fluence of $0.571 \mathrm{~J} / \mathrm{cm}^{2}$, still below the damage threshold. For the $20 \mathrm{~mm}$ crystal by contrast, damage was observed at a lower threshold than expected, and limited the efficiency to $0.13 \%$ at a pump fluence of $0.287 \mathrm{~J} / \mathrm{cm}^{2}$, where saturation had not yet occurred.

A record multi-cycle $\mathrm{THz}$ energy of $40 \mu \mathrm{J}$ was achieved at a frequency of $0.544 \mathrm{THz}$, using the $10 \mathrm{~mm}, 212 \mu \mathrm{m}$-poled PPLN crystal (with an aperture of $3 \mathrm{~mm} \times 10 \mathrm{~mm}$ ) at cryogenic temperature. Although the $20 \mathrm{~mm}$ piece exhibited a higher peak efficiency, the energy output was limited to $12.8 \mu \mathrm{J}$ by a smaller aperture of $3 \mathrm{~mm} \times 3 \mathrm{~mm}$.

The transfer of energy between the optical and $\mathrm{THz}$ fields necessarily results in a modification of the optical spectrum, providing another means of diagnosing the nonlinear process. For instance, conservation of energy dictates that the centroid shift of the optical spectrum represents an upper limit to the conversion efficiency. In addition, for efficient terahertz generation an energy transfer process known as cascading is required to circumvent the Manley-Rowe limit [19]. During cascading, optical photons interact nonlinearly multiple times, producing multiple $\mathrm{THz}$ photons for each optical one. This process can result in both red- and blue-shifting of these photons by multiple times the frequency of the $\mathrm{THz}$ photons. These effects are illustrated in figure 5. The transmitted optical spectrum shows an overall red-shift of the centroid from $798.1 \mathrm{~nm}$ before the interaction to $801.4 \mathrm{~nm}$ after the interaction, corresponding to an energy loss of $0.41 \%$. The difference spectrum (shaded gray) shows evidence of the cascading process since the red-and blue-shifts of $\sim 20 \mathrm{~nm}$ correspond to roughly $17 \times$ the $\mathrm{THz}$ photon energy.

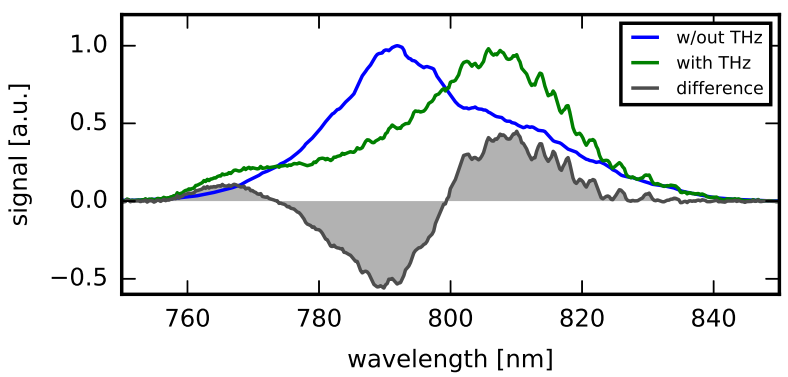

Fig. 5. Transmitted pump spectra before (blue) and after interaction with the $212 \mu \mathrm{m}$ PPLN (green). The shadowed graph demonstrates an energy transfer due to $\mathrm{THz}$ generation. For this measurement the crystal was cooled to $82 \mathrm{~K}$ and operated with a pump fluence of $F=0.287 \mathrm{~J} / \mathrm{cm}^{2}$.

The proof-of-principle results demonstrated here can be improved upon in several ways. Of particular importance is implementation of an AR coating to reduce the $\sim 44 \%$ loss to the extracted $\mathrm{THz}$ energy from Fresnel reflections at the crystal exit face. In addition, the small amount nonlinear chirp imparted to the optical beam by the ANGUS stretcher is estimated to induce a frequency difference that varies over the spectrum of the two chirped pulses such that only a portion of the pump pulses are phase-matched to the $\mathrm{THz}$ frequency. Compensating for the higher order dispersion in the pump pulse, we estimate a significantly increased conversion efficiency, approaching the $1 \%$ level for a $212 \mu \mathrm{m}$-poled PPLN crystal [17]. Finally, by increasing the crystal aperture sizes to the maximum that is currently possible [20], it may be possible to increase pump energies to the Joule level and take full advantage of the capabilities of current Ti:Sa laser systems. Chirp-and-delay pumping of PPLN is thus a promising scheme for generating narrowband $\mathrm{THz}$ pulses at the $\mathrm{mJ}$ level required by future applications.

We thank V. Leroux and M. Schnepp for operating the ANGUS laser and M. Schust, T. Tilp, A. Berg, and J. Derksen for technical support on the experimental setup. This work has been supported by the European Research Council under the European Union Seventh Framework Program (FP7/2007-2013)/ ERC Grant Agreement no. 609920 - AXSIS and the project ELI - Extreme Light Infrastructure - phase 2 (CZ.02.1.01/0.0/0.0/15_008/0000162) from European Regional Development Fund.

\section{REFERENCES}

1. J. Hebling, K. L. Yeh, M. C. Hoffmann, and K. A. Nelson, IEEE Journal of Selected Topics in Quantum Electronics 14, 345 (2008).

2. E. A. Nanni, W. R. Huang, K.-H. Hong, K. Ravi, A. Fallahi, G. Moriena, R. J. D. Miller, and F. X. Kärtner, Nature communications 6 (2015).

3. T. Kampfrath, K. Tanaka, and K. A. Nelson, Nat Photon 7, 680 (2013).

4. A. G. Stepanov, J. Hebling, and J. Kuhl, Applied Physics Letters 83, 3000 (2003).

5. M. Beck, I. Rousseau, M. Klammer, P. Leiderer, M. Mittendorff, S. Winnerl, M. Helm, G. N. Gol'tsman, and J. Demsar, Phys. Rev. Lett. 110, 267003 (2013).

6. Y.-S. Lee, T. Meade, V. Perlin, H. Winful, T. B. Norris, and A. Galvanauskas, Applied Physics Letters 76, 2505 (2000).

7. C. Weiss, G. Torosyan, Y. Avetisyan, and R. Beigang, Opt. Lett. 26, 563 (2001).

8. K. L. Vodopyanov, Optics Express 14, 2263 (2006).

9. S. Carbajo, J. Schulte, X. Wu, K. Ravi, D. N. Schimpf, and F. Kärtner, Optics Letters 40, 5762 (2015).

10. K. Ravi, D. N. Schimpf, and F. X. Kärtner, Opt. Express 24, 25582 (2016).

11. A. S. Weling, B. B. Hu, N. M. Froberg, and D. H. Auston, Applied Physics Letters 64, 137 (1994).

12. Z. Chen, X. Zhou, C. A. Werley, and K. A. Nelson, Applied Physics Letters 99, 071102 (2011).

13. M. M. Fejer, G. A. Magel, D. H. Jundt, and R. L. Byer, IEEE Journal of Quantum Electronics 28, 2631 (1992).

14. M. Yamada, N. Nada, M. Saitoh, and K. Watanabe, Applied Physics Letters 62, 435 (1993).

15. N. E. Yu, K. S. Lee, D.-K. Ko, C. Kang, S. Takekawa, and K. Kitamura, Optics Communications 284, 1395 (2011).

16. Z. Cao, X. Gao, W. Chen, H. Wang, W. Zhang, and Z. Gong, Optics and Lasers in Engineering 47, 589 (2009).

17. S. W. Jolly, F. Ahr, N. H. Matlis, F. X. Kärtner, and A. R. Maier, in preparation (2017).

18. X. Wu, C. Zhou, W. R. Huang, F. Ahr, and F. Kärtner, Optics Express 23, 29729 (2015).

19. M. Cronin-Golomb, Opt. Lett. 29, 2046 (2004).

20. H. Ishizuki and T. Taira, Opt. Express 20, 20002 (2012). 